\title{
A Mental Disorder Prediction Model with the Ability of Deep Information Expression Using Convolution Neural Networks Technology
}

\author{
Pufang Huang \\ Shenzhen Institute of Information Technology, Shenzhen, Guangdong 518172, China \\ Correspondence should be addressed to Pufang Huang; 2017000022@sziit.edu.cn
}

Received 27 November 2021; Revised 16 December 2021; Accepted 17 December 2021; Published 31 January 2022

Academic Editor: Tongguang $\mathrm{Ni}$

Copyright (c) 2022 Pufang Huang. This is an open access article distributed under the Creative Commons Attribution License, which permits unrestricted use, distribution, and reproduction in any medium, provided the original work is properly cited.

\begin{abstract}
The psychological health education work of all universities is currently facing the same problem, despite the rapidly expanding scale of universities and the ever-increasing demand for students: there are few teachers engaged in this field, who cannot meet the urgent needs of the majority of students. Because of their mental illness, university students are unable to complete their studies in a timely manner, affecting their own development. One after the other, such occurrences occur. This paper uses the common mental disorder identification of university students as an example and builds a mental disorder identification model based on CNN. The model has the ability to learn on its own, allowing it to diagnose psychological disorders in university students and provide support for college psychological counselling and the psychological health team. However, because China currently pays little attention to the psychological health of this population, it is necessary to investigate and analyse the psychological health of university students at this time.
\end{abstract}

\section{Introduction}

Many people are experiencing varying degrees of psychological barriers as a result of increasing pressure from all directions in society, family, and life. Social stability, family harmony, and happiness have all suffered as a result of psychological obstacles [1]. The psychological health education work of all universities is currently facing the same problem, despite the rapidly expanding scale of universities and the ever-increasing demand for students: there are few teachers engaged in this field, which cannot meet the urgent needs of the majority of students [2]. The severe shortage of full-time psychological counsellors in universities has become a major impediment to psychological health education and intervention. According to the analysis of the factors affecting the psychological health of contemporary university students, we can summarize it into 10 influencing factors: personal future pressure, social pressure, economic pressure, environmental adaptation pressure, study pressure, emotional pressure, parental expectation pressure, family influence pressure, professional emotional pressure, and interpersonal pressure. We must take seriously the psychological health problems of university students, fully analyse and study the causes of university students' mental problems, and understand the factors affecting psychological health. Starting at the source, using effective approaches to detect early warning indications of psychological issues in order to reach the goal of prevention, to truly solve the problem of university students' psychological health, suffocate the emergence of psychological problems in the cradle. The development of artificial intelligence theory brings opportunities to computer-aided mental disorder identification [3]. The modeling and prediction of psychological disorders can help relevant experts and scholars understand the changing characteristics of psychological disorders and formulate relevant treatment plans for patients with psychological disorders. Therefore, the prediction of psychological disorders has very important social and practical application value.

People used experts to predict psychological disorders at first. In this method, the predicted outcomes of 
psychological disorders are insecure. Different experts can produce different forecast outcomes, and forecast outcomes are closely related to expert knowledge, making psychological disorder forecast outcomes highly subjective and unreliable [4]. They are exceptional young college students who represent the educated youth [5]. Many studies in the literature have shown that NN can effectively model and predict time series. This paper analyses the reasons affecting the psychological health of Chinese university students, expands the evaluation factors, collects the corresponding data, uses the convolution $\mathrm{NN}$ model to predict and analyse the psychological health of university students, and establishes a convolution NN forecasting model suitable for the identification of mental disorders, based on the current situation and characteristics of Chinese students.

Currently, universities only offer consultation and guidance in the field of psychological health education to university students through school psychological counselling teachers or hospital psychological experts. However, due to varying levels of experience and skill, it is unable to produce the desired effect. The NN expert system can perform complex pattern recognition and tasks with complex rules that cannot be predicted ahead of time. They are under significantly more psychological stress than their peers. As a result, research into the psychological well-being of today's university students is critical. In order to cope with psychological health demands in the future, researchers are looking into the internal law of numerous aspects affecting psychological health. This helps to generate new ideas for psychological health work. This paper developed a CNN-based [6] psychological disorder identification model. The error between the predicted outcomes and the measured value output by the ANN [7] is small after sample training, and the expected effect is well achieved. The model can detect university students' psychological status and provide diagnosis results in real time, as well as providing support for psychological consultation and psychological health teams in universities.

\section{Related Work}

Psychological health education in foreign universities is divided into three stages, according to literature [8]: initial stage, development stage, and mature stage. According to statistics in literature [9], university students who have dropped out of school due to various mental disorders caused by mental illness account for about half of all dropouts. Literature [10] combines knowledge discovery and NNs to produce forecast results that outperform traditional methods. A survey of 140 medical graduate students was conducted in literature [11]. It was discovered that the range values of various factors among major students differ significantly from the national average and that some factor scores are lower than those of ordinary people. The single factor analysis method is used to analyse related factors in literature [12] in the research of postgraduates' physical and mental symptoms. Social factors such as gender, whether they are an only child, and personality traits have been shown to influence graduate students' physical and mental symptoms in studies. Literature [13] surveyed postgraduates, showing that the psychological health of female postgraduates is significantly higher than that of male postgraduates in terms of both factor scores and the total score of the scale. In literature [14, 15], with the help of the idea of quadratic performance index in optimal control, it is proposed to use quadratic performance index to calculate the control law to obtain the desired optimization effect. Literature [16] mentioned that, with social changes and the influx of only children into universities, the number of university students suffering from psychological disorders had shown a clear upward trend. Literature [17] conducted a survey of 400 medical graduate students in Chengdu and found that the suicide probability of girls was $78.99 \%$, while that of boys was only $58.60 \%$. In literature [18], a survey of 289 medical master's students in a military school was grouped by gender, and the grouping results were compared with several domestic research results. The comparison shows that female students are higher than male students in some factors. Literature [19] proposed a hybrid learning algorithm that alternately uses a genetic algorithm and $\mathrm{CNN}$ algorithm. In literature [20], 828 postgraduates on campus are taken as the research objects regarding the personality differences of graduate students and the differences in their living conditions. Through discussion, it is found that some factors of the graduate student population are higher than the national norm range value. Due to the differences in the personality of this group, psychological health presents the phenomenon of "three highs" and "three lows." Literature [21] proposes to combine multiple population genetic algorithms with NNs, which can simultaneously consider global optimization solutions and local optimization solutions. Literature [22] uses NNs to establish mathematical models to solve practical problems. Literature [23] first applied the support vector machine model to time series forecasting in 1997. Literature [24] verifies the prediction effect of support vector machines on five financial time series data. The experimental results show that the evaluation indicators such as the prediction mean square error and the average absolute error on the five data samples of the support vector machine are better than the backpropagation NN. Literature [25] survey on university students across the country shows that $22.01 \%$ of the students have obvious psychological problems, and about $3 /$ 8 of the students have different degrees of psychological obstacles. Literature [26] proposed an NN forecasting model with lower complexity in 2009. In this paper, according to the current psychological health status of university students, a prediction system model of psychological health status of university students is established based on CNN. This model has high precision and high efficiency. Students' psychological problems can be predicted in advance, and corresponding measures can be taken in time. Finally, the stability and unity of the school and the growth of students will be realized.

\section{Mental Disorder Prediction Based on CNN}

3.1. NN Algorithm. In the field of computer science and artificial intelligence, artificial neural network (ANN) simulates the information dissemination mechanism of the 
biological nervous system so that the machine can imitate the human brain to learn and recognize information [27]. NNs perform calculations and spread information through a large number of interconnected neurons. They are commonly used to describe the complex relationship between input and output, as well as investigate data's internal structure and patterns. The topological structure of ANNs' connections, as well as the strength of synaptic connections or connection weights, determines their functional characteristics. A matrix $\mathrm{W}$ can be used to represent the weights of all NN connections. Its entirety reflects the NN's knowledge storage for the problem at hand. Through the learning and training of samples, the $\mathrm{NN}$ can continuously change the connection weight and topology of the network, bringing the network's output closer to the desired output. The dynamic adjustment of variable weights is the essence of this process, which is known as NN learning or training.

Artificial neuron is a simulation of the characteristics of weighted summation of the input information of biological neuron. It uses the activation function to map the obtained network input to realize the processing of amplifying or suppressing the output value of the neuron. Assuming that the $n$ inputs of a neuron are represented by $x_{1}, x_{2}, \ldots, x_{n}$, the connection weight corresponding to each input is $w_{1}, w_{2}, \ldots, w_{n}$, the threshold is represented by $\theta$, and the actual output value of the neuron is represented by $y$, then the input-output mapping relationship can be represented by the following formula:

$$
y=f\left(\sum_{j=1}^{n} w_{j} x_{j}-\theta\right) .
$$

Here, $f$ is called the activation function. Figure 1 shows the structure of a single artificial neuron computer system.

The learning method of $\mathrm{CNN}$ is the third major factor that determines the information processing performance of $\mathrm{CNN}$, so the study of learning plays an important role in $\mathrm{NN}$ research [28]. Rules that change the weights are called learning rules or learning algorithms. When a single processing unit collectively adjusts the weights, the network shows "intelligent" characteristics. Meaningful information is distributed and stored in the adjusted weight matrix.

3.2. The Evaluation Model of University Students' Psychological Health Status. The preparation of the data shows a very positive role in ANN. Good data can quickly balance the convergence of the network, and it is close to the output result. On the contrary, bad data, no matter how to modify the network parameters, cannot make the network achieve the required effect. Taking the identification of common psychological diseases of university students from five characteristics as an example, the development process of model service is illustrated. The five characteristics of input include behaviour, emotional state, diet and sleep, personality characteristics, and physical diseases. Four common mental diseases of university students are selected as training, and an $\mathrm{NN}$ for identifying mental diseases is constructed. In practice, the features of mental illness are

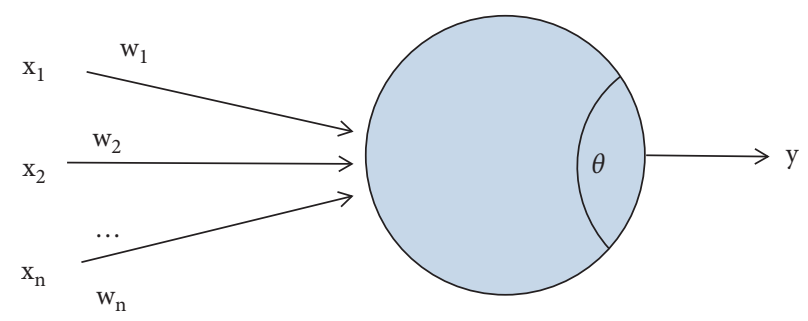

FIgURE 1: Artificial neuron.

much more complicated and huge, and there are many overlapping interactions among the features. Here, only five distinctive commonalities are selected to construct the training set of $\mathrm{NN}$ recognition.

The improved NN psychological barrier forecasting model works on the following principle. To begin, gather historical data on psychological barriers. The chaos algorithm is then used to preprocess historical data on psychological disorders in order to better understand how their characteristics change over time. Finally, NN is used to learn preprocessed historical data of psychological disorder, and the particle swarm optimization algorithm is used to improve NN's existing problems and establish the best psychological disorder forecasting model. Figure 2 depicts the working principle of the improved NN-based mental disorder forecasting model.

The period in which college students live is a critical transition period for a person to mature. During this period, they will face many problems such as emotion and going to the society. If not handled properly, various psychological problems will appear, such as depression and anxiety. Convolution layer uses local connection and weight sharing to reduce the number of network-free parameters and the complexity of network parameters. The convolution layer calculation formula is as follows:

$$
X^{(l)}=f\left(W^{l} \otimes X^{(l-1)}+b^{(l)}\right) .
$$

Here, $X^{(l)}$ and $X^{(l-1)}$ represent the neuron activity of layer $l$ and $1-1, W^{l}$ represents the convolution kernel, and $b$ represents the bias.

In order to make the training of NN more effective and improve the training speed of the established $\mathrm{NN}$, the input and output data of NN should be preprocessed first. For NN calculation, it is necessary to convert literal concepts into numerical values. In order to facilitate the discrimination of data, six-dimensional vector values are used to represent each feature. The first three bits represent categories, and the last three bits represent features, so a total of $26=64$ features can be accommodated. The outputs of the four subconvolution networks are fused in the full connection layer. The dimensions of the first full connection layer and the second full connection layer are 512 and 256, respectively. Finally, the Softmax function is selected as the output classifier. The Softmax function estimates the probability that input $x$ belongs to a specific category $j \in \mathrm{k}$ :

$$
P(y=j \mid x)=\frac{\exp \left(x^{T} W_{j}\right)}{\sum_{k=1}^{K} \exp \left(x^{T} W_{k}\right)} .
$$




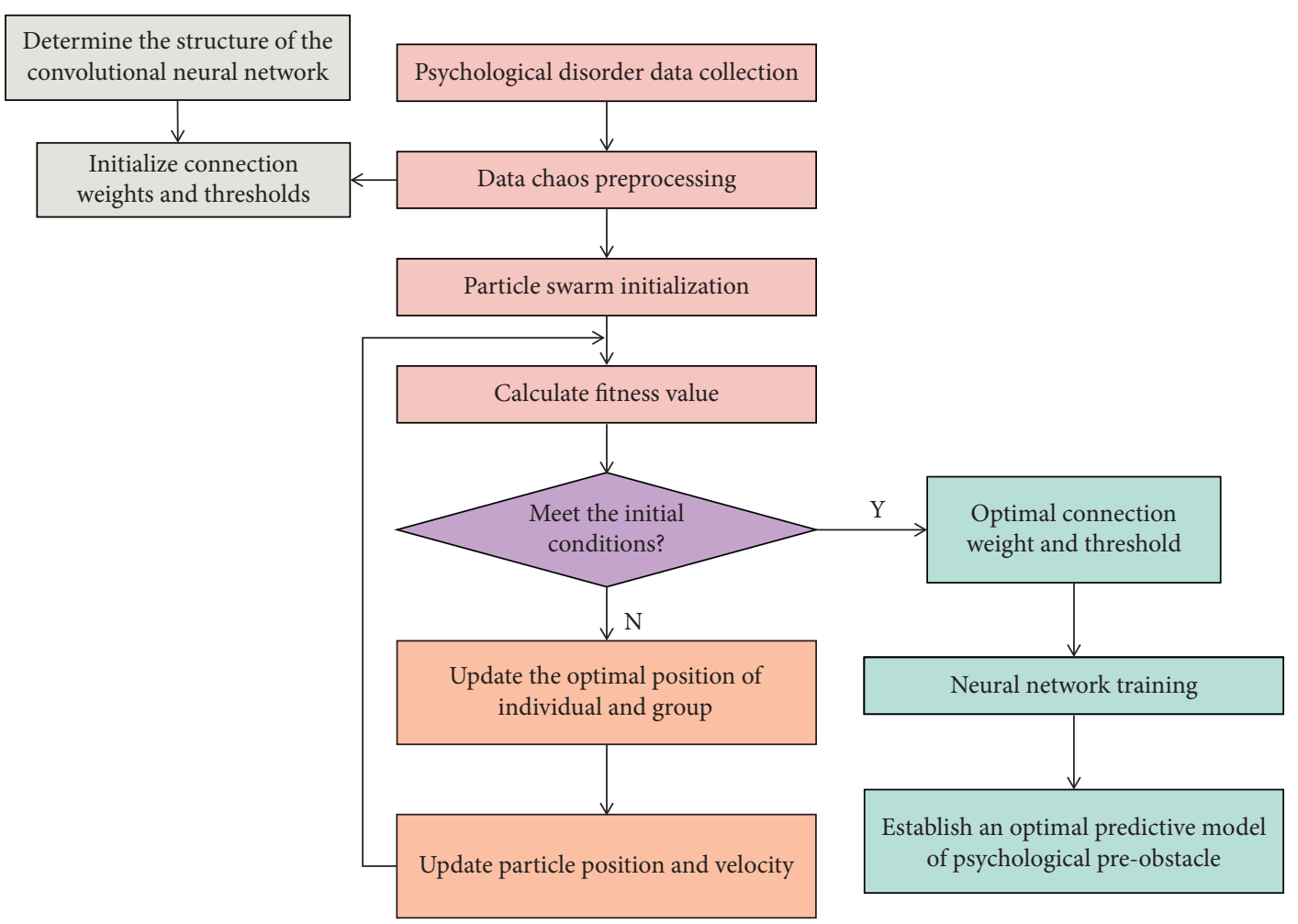

FIGURE 2: The working principle of the psychological disorder forecasting model of the improved NN.

Select the commonly used Rectified Linear Units (ReLU) as the excitation function. The ReLU excitation function can prevent gradient disappearance and overfitting. The ReLU excitation function is defined as

$$
f_{\text {cov }}(x)=\max (0, x) \text {. }
$$

Dropout is an effective means to prevent overfitting and improve the effect in CNN. The feature representation of data is very important to the successful training of the model. Better data representation can reduce or even eliminate the influence of factors unrelated to the training task in the original data on the training results and save useful information that is beneficial to the training task.

The characteristics of each influencing factor are sorted according to the degree of psychological resistance, the index of the category affected by each influencing factor in the initial mental performance state is obtained, and the mental performance state of different influencing categories is obtained. For m-dimensional college students' psychological complexity, the total number of different mental performance state sequences is $m$, the probability of $k$ different mental performance state sequences is assumed to be $P_{1}, P_{2}, \ldots, P_{k}$, and the probability of the mental state sequence is ranked. Define permutation entropy:

$$
H_{P E}(m)=-j=1 \sum^{k} P_{j} \operatorname{In} P_{j}
$$

Integrate the state sequence given by (5), and use (6) to express the emotional characteristics of behaviour corresponding to the mental state at this stage:

$$
0 \leq H_{P E}=\frac{H_{P E}}{\operatorname{In}(m)} \leq 1 .
$$

Suppose that $\{x(t), t=1,2, \ldots, N\}$ represents the time series set of behavioural emotional characteristics of college students' mental state. Due to the complexity of the behavioural tendency of college students dominated by psychology, it is necessary to construct a relationship with the psychological duration $\tau$, select the duration sequence $x(t+\tau)$ to form a new duration point sequence $y(t)$, and determine it according to the correlation between the calculation of $x(t)$ and $y(t)$ and psychological duration $\tau$.

This paper uses the prevalence of mental disorders as historical data of mental disorders. They compose a kind of time series data according to the chronological order, without considering related influencing factors, so that the historical data composes a one-dimensional time series data. In order to obtain a higher-precision mental disorder prediction structure, an improved NN mental disorder forecasting model is proposed. This model has the ability of autonomous learning and can realize the diagnosis of university students' psychological disorders.

\section{Result Analysis and Discussion}

Researchers simulated the computing power of real NN to create NN. Because it gradually demonstrates powerful functions such as learning, memory, and association during its development, $\mathrm{NN}$ is widely used in a variety of fields. Simple pattern recognition is realized through NN application research in the diagnosis of university students' 
psychological disorders, and the expected diagnosis effect is achieved. Currently, the system is just a simple simulation of a specific biological nervous system performance. The system can theoretically perform complex pattern recognition and complete tasks with complex rules that cannot be determined in advance if enough training and learning are done. The internal representations used by the neural network after learning the sample set are the weights and thresholds that can best meet the data requirements obtained by the neural network through adaptive learning. The feature data from the sample set to be identified is then fed into the trained network, which can then reason and identify the sample output results automatically. When viewed as a mapping from input data to output data, the neural network is highly nonlinear. Figures 3-6 show the results of the experiments.

It can be seen that no matter whether the input sample length is 20 or 30 when the convolution layer and downsampling layer are 1, the root mean square error and correlation coefficient of the forecast outcomes are better than when the convolution layer and downsampling layer are 2 . The determination coefficient of the forecast outcomes is closer to 1 . In this paper, it is considered that it is more reasonable to choose 1 for convolution layer and downsampling layer of convolution $\mathrm{NN}$ exchange rate forecasting model.

According to the degree of psychological resistance, the characteristics of each influencing factor are sorted, the indicators of the categories affected by each influencing factor under the initial mental performance state are obtained, and the mental performance states of different influencing categories are obtained. A single neuron can only perform very simple functions. If you want to realize the functional simulation of the human brain $\mathrm{NN}$ and complete complex tasks, you need to connect a large number of neurons in a certain way to form an ANN with different topological structures. We use the $\operatorname{train}()$ function to train the CNN model. The error curve after training is shown in Figure 7.

According to the graph description, it can be seen that, during the self-learning process of the network, the error is constantly decreasing, and the error basically reaches the target at Step 780. Through the continuous training of NN, the network can basically achieve the minimum error prediction. It can be seen that the forecasting model has a good prediction effect. Through the continuous training of $\mathrm{NN}$, the network can basically achieve the minimum error prediction and thus can meet our needs. The comparison curve between the forecast outcomes and the true value for 2020 is shown in Figure 8.

Through the simulation test of several groups of data, it can be seen that the error between the predicted results and the true values of each group is very small, and the fitting degree of the input data basically meets the required requirements. It shows that the established model has certain accuracy and can fully predict the psychological health of university students.

The same parameters are used for the three subconvolution networks with dynamic input data. To extract the characteristics of each user's behaviour at different time

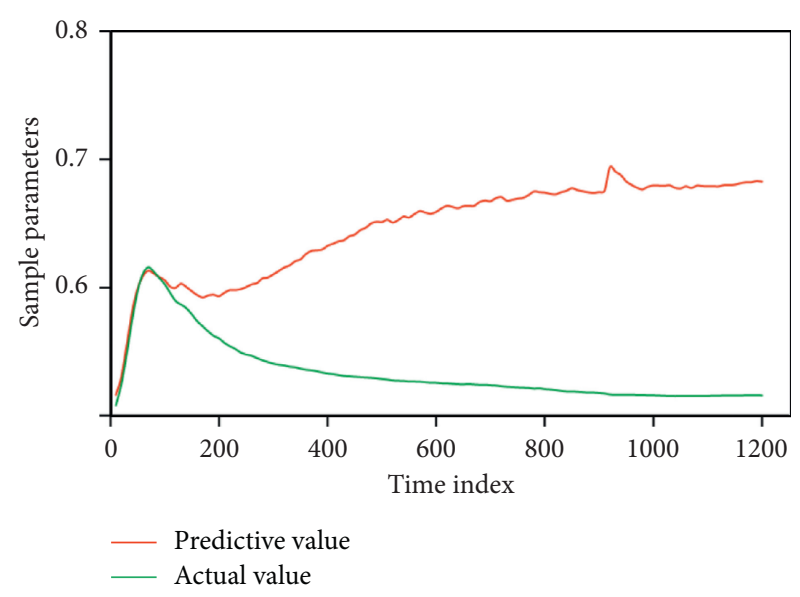

Figure 3: The forecast outcomes with the input sample length being 20 and the number of convolutional layers being 1 .

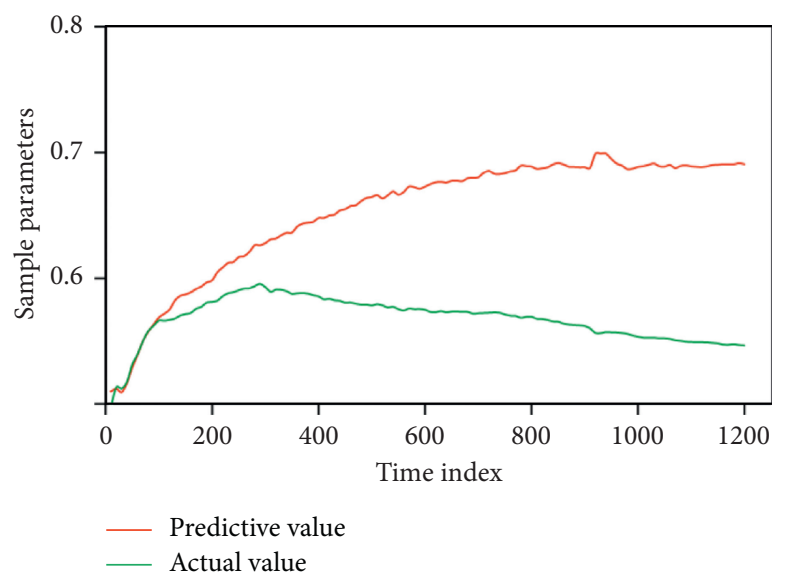

Figure 4: The forecast outcomes with the input sample length being 20 and the number of convolutional layers being 2 .

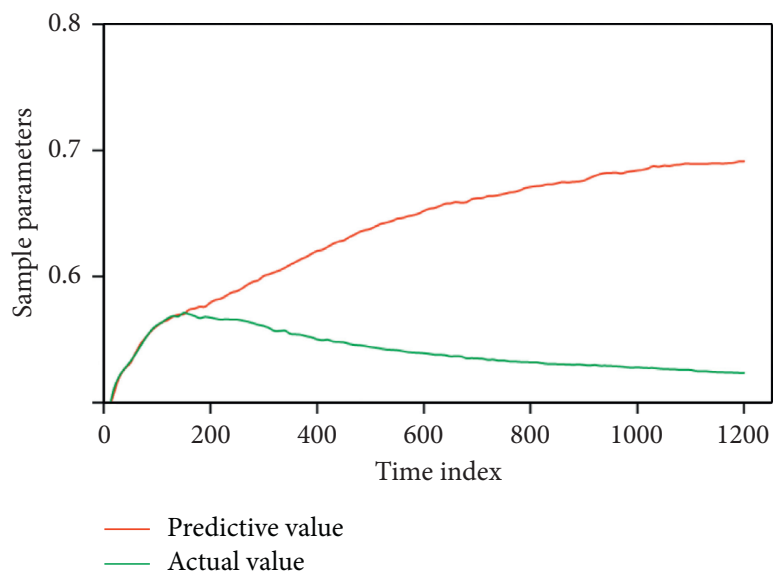

FIgURE 5: The forecast outcomes with the input sample length being 30 and the number of convolutional layers being 1 .

points, the first convolution layer selects 64 convolution kernels with a size of 13 . Following the convolution layer is the pooling layer, which is responsible for secondary feature 


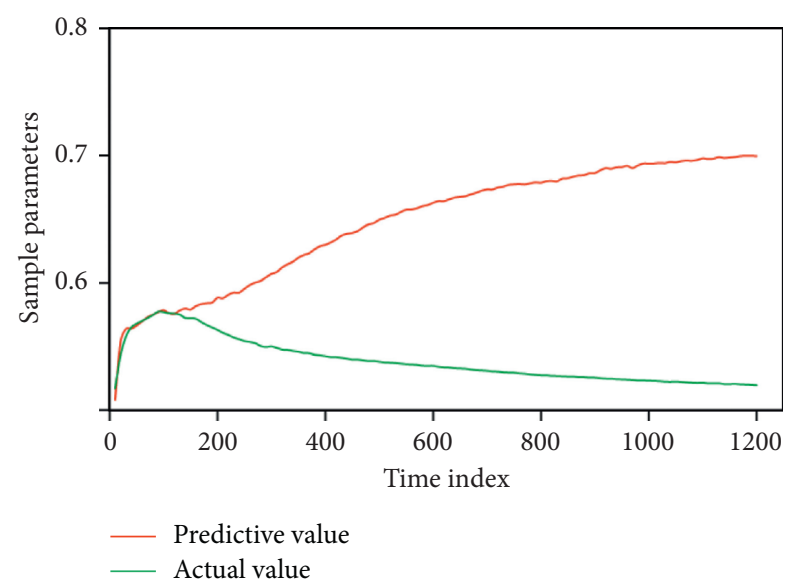

FIgURE 6: The forecast outcomes with the input sample length being 30 and the number of convolutional layers being 2 .

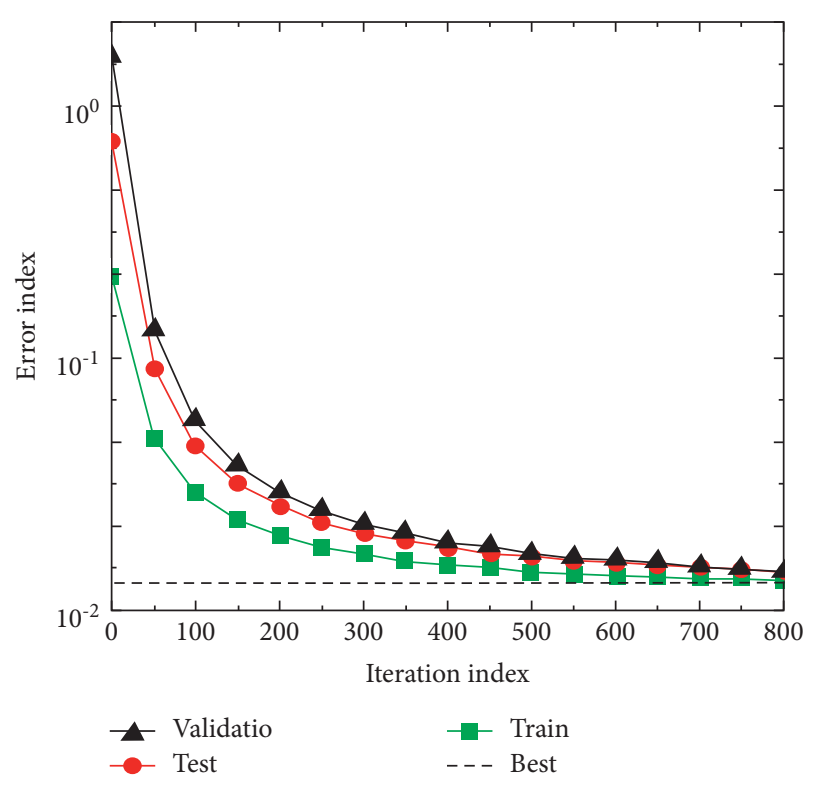

FIGURE 7: NN training process.

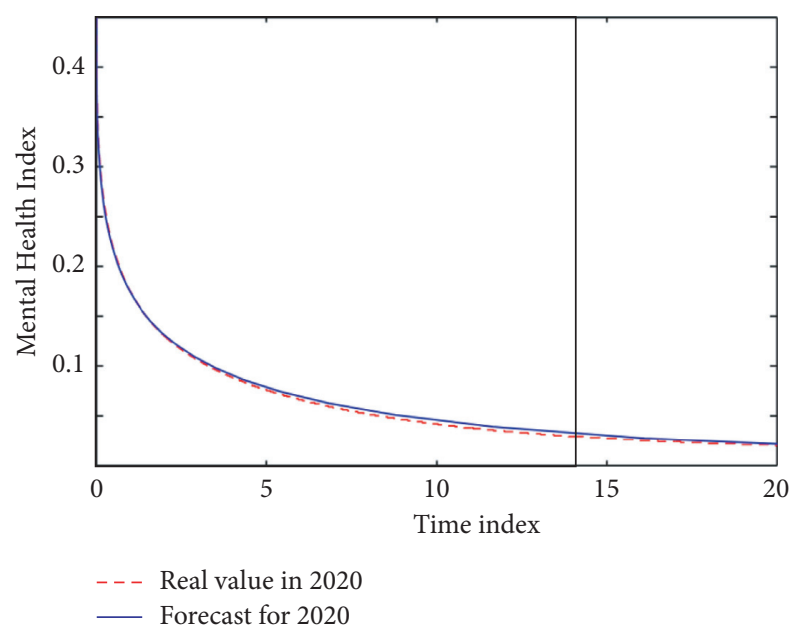

FIGURE 8: Comparison between predicted and real values of psychological health factors in 2020.

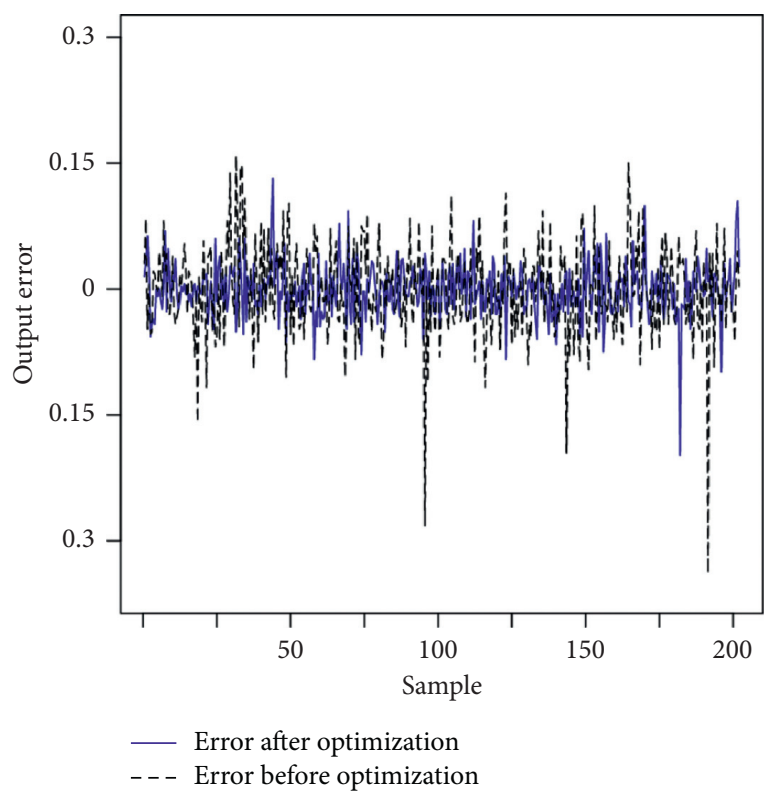

FIgURE 9: Error comparison before and after optimization.

extraction. Choose the most efficient pooling method. After the pooling layer, the second convolution layer is connected, and 128 convolution kernels with a size of 33 are chosen. The goal is to extract the characteristics of each user behaviour index at various time points, as well as the characteristics of multiple user behaviours at the same time. After the second convolution layer, a pooling layer is added. A convolution layer and a pooling layer are used to extract features from static input data. The convolution layer employs 6413 -size convolution kernels. Figure 9 shows the error comparison before and after the algorithm optimization.

It can be seen from Figure 9 that, by comparing the errors before and after optimization with a genetic algorithm, it can be found that the optimized NN forecasting model is better than that before optimization, and the optimized NN can achieve the data fitting effect faster.

Predicting the mental health of college students is a complex and systematic process. Because the process by which each specific factor performs its function is invisible and intangible and because the influence on the outcome may be nonlinear, a method that can reflect the corresponding relationship between the factors that affect the psychological state and the outcome is required to develop the prediction model. The ability of the optimized NN to fit data is clearly improved without changing the original network structure and network parameters. The reason for this is that the genetic algorithm uses the fitness function to determine the optimal weights and thresholds and then passes those weights and thresholds to NN training to replace the random weights and thresholds generated by Matlab in each training. This severely limits the ability to adjust the weight threshold on one's own. The number of iterative steps in the $\mathrm{NN}$ is significantly reduced after optimization, and the preset goal can be reached much faster. Experiments show that using a genetic algorithm improves the initial weights and thresholds of $\mathrm{CNN}$, allowing it to fit the data better. 
The ability of fuzzy clustering analysis and prediction of optimized mental health data is obviously improved because $\mathrm{CNN}$ finds out the optimal weights and thresholds through fitness function and then gives the weights and thresholds to neural network training to replace the initial weights and thresholds randomly generated by Matlab in each training, which greatly reduces the ability of self-adjustment of weights and thresholds. By changing the parameters of the $\mathrm{NN}$, the calculation of the final result of the prediction system is simple and flexible, and the prediction efficiency and accuracy of the network model are greatly improved. This provides a good reference method for the study of university students' psychological health to a great extent.

\section{Conclusions}

The significance of psychological health among university students is self-evident. It has a huge impact on university students, schools, society, and the country as a whole. Nowadays, the pace of university students' studies and lives is quickening, and social influence is growing, as well as psychological pressure on students. In terms of psychology, university students experience a variety of negative reactions and maladjustment. Some of them are downright dangerous. Suspension from school, dropping out, and even wounding and suicide as a result of mental illness have all increased in recent years. Psychological factors are the primary cause of psychological illnesses. Psychological issues have a significant impact on university students' development. As a result, universities have made it one of their top priorities to prioritise psychological health education for university students. The psychological well-being of university students has recently become a hot topic. The ability of fuzzy clustering analysis and prediction of optimized mental health data is clearly improved because CNN finds the optimal weights and thresholds through fitness function and then gives the weights and thresholds to neural network training to replace the initial weights and thresholds randomly generated by Matlab in each training, greatly reducing the ability of weights and thresholds self-adjustment. As a result, this paper builds a forecasting model of university students' psychological disorders based on CNN. The results show that the improved $\mathrm{NN}$ overcomes the drawbacks of the current mental disorder forecasting model, allowing university students to know their psychological health status in real time. It is a high-precision, high-efficiency model for predicting psychological disorders with a wide range of applications. We can predict students who may have psychological problems in advance using this $\mathrm{NN}$ psychological forecasting model, so that appropriate attention or measures can be provided on time, avoiding the enlargement or aggravation of students' psychological problems and ensuring the positive and stable development of schools and students.

\section{Data Availability}

The data used to support the findings of this study are included within the article.

\section{Conflicts of Interest}

The author declares no conflicts of interest.

\section{Acknowledgments}

The study was supported by the Annual Psychologic Health Program (Project no. HX-239).

\section{References}

[1] Y. Chen, H. Jiang, C. Li, X. Jia, and P. Ghamisi, “Deep feature extraction and classification of hyperspectral images based on convolutional neural networks," IEEE Transactions on Geoscience and Remote Sensing, vol. 54, no. 10, pp. 6232-6251, 2016.

[2] L. Gao, J. Wang, Y. Fan, and C. Niannian, "Robust visual tracking based on convolutional neural network and consistency predictor," Acta Optics, vol. 37, no. 8, p. 10, 2017.

[3] J. Liu, R. Cui, and Y. Zhao, "Cross-language sentiment classification based on self-associative memory and convolutional neural network," Journal of Chinese Information Processing, vol. 032, no. 012, pp. 118-124, 2018.

[4] Z. Shen and S. Yuan, "Regional load clustering integrated forecasting using convolutional neural network support vector regression machine[J]," Power System Technology, vol. 44, no. 6, 8 pages, 2020.

[5] X. Chen, J. Yi, and J. Dai, "Convolutional neural network radiotherapy plan dose prediction: comparison of two decoders[J]," Chinese Journal of Medical Physics, vol. 37, no. 2, 6 pages, 2020.

[6] M. Li, G. Zhou, W. Cai et al., "Multi-scale sparse network with cross-attention mechanism for image-based butterflies finegrained classification," Applied Soft Computing, Article ID 108419, 2022, In Press.

[7] Z. Huang, Y. Liu, C. Zhan, C. Lin, W. Cai, and Y. Chen, “A novel group recommendation model with two-stage deep learning," IEEE Transactions on Systems, Man, and Cybernetics: Systems, In Press, 2021.

[8] Z. Su, Y. Kang, B. Zhang, Z. Zhang, and H. Jiang, "Disorder induced phase transition in magnetic higher-order topological insulator: a machine learning study[J]," Chinese Physics B, vol. 28, no. 11, pp. 312-319, 2019.

[9] X. Song, G. Ye, J. Zhou, and F. Li, "Prediction of effective diffusion coefficient of porous materials based on convolutional neural network," Chemical Reaction Engineering and Technology, vol. 34, no. 2, pp. 223-240, 2018.

[10] S. Wang, J. Ma, and J. Xu, “AUCpreD: proteome-level protein disorder prediction by AUC-maximized deep convolutional neural fields," Bioinformatics, vol. 32, no. 17, pp. 672-679, 2016.

[11] H. Chang, X. Chen, A. Zhang, Ce Li, and D. Lin, "Convolutional Neural Network Embedding Improved SENet for Continuous Blood Pressure prediction," Computer Engineering and Applications, pp. 2130-135, 2021.

[12] Y. Xu, Z. Wu, H. Zhu, B. Wang, and Z. Deng, "Short-term power load forecasting based on multi-scale convolutional neural network," Journal of Shenyang University of Technology, vol. 42, no. 6, 6 pages, 2020.

[13] X. Li, J. Wang, and A. Li, "Progress in imaging studies of convolutional neural networks in mild cognitive impairment [J]," Magnetic Resonance Imaging, vol. 12, no. 9, 4 pages, 2021. 
[14] A. Shafiee, A. Nag, N. Muralimanohar et al., "Isaac," $A C M$ SIGARCH - Computer Architecture News, vol. 44, no. 3, pp. 14-26, 2016.

[15] M. Jaderberg, K. Simonyan, A. Vedaldi, and A. Zisserman, "Reading text in the wild with convolutional neural networks," International Journal of Computer Vision, vol. 116, no. 1, pp. 1-20, 2016.

[16] H. Zhang, Z. Liu, and H. Li, "Time series prediction method of production process based on associated variable time lag analysis convolutional neural network," CIE Journal, vol. 68, no. 9, 10 pages, 2017.

[17] Y. Liu, L. Zhu, and Y. Zhou, "Convolutional neural network and its application in ore prospecting prediction: taking Zhaojikou lead-zinc deposit in Anhui Province as an example," Acta Petrologica Sinica, vol. 34, no. 11, 8 pages, 2018.

[18] A. Saeedi, M. Saeedi, A. Maghsoudi, and A. Shalbaf, "Major depressive disorder diagnosis based on effective connectivity in EEG signals: a convolutional neural network and long short-term memory approach," Cognitive Neurodynamics, vol. 15, no. 2, pp. 239-252, 2021.

[19] N. Lin, D. Zhang, K. Zhang, and M. Li, "Small sample convolutional neural network learning and prediction of seismic oil and gas reservoirs," Chinese Journal of Geophysics, vol. 61, no. 10, pp. 75-86, 2018.

[20] S. Yang, R. Wang, and Y. Wang, "Soil organic carbon prediction model based on convolutional neural network and near-infrared spectroscopy," Computer Applications and Software, vol. 35, no. 10, 7 pages, 2018.

[21] M. Zeng, X. Yang, and Q. Zhu, "Lateral rotation angle prediction method based on convolutional neural network," Modern Electronic Technology, vol. 43, no. 6, 4 pages, 2020.

[22] H. Zhang, C. Huang, S. Tang, and X. Yongbo, "Real-time prediction of unmanned combat aircraft flight trajectory based on convolutional neural network," Acta Armamentarii, vol. 41, no. 9, 10 pages, 2020.

[23] S. Chen and X. Yang, "Short-term bus passenger flow prediction based on improved convolutional neural network," Computer Science, vol. 046, no. 005, pp. 175-184, 2019.

[24] D. Arifoglu and A. Bouchachia, "Detection of abnormal behaviour for dementia sufferers using Convolutional Neural Networks," Artificial Intelligence in Medicine, vol. 94, no. 3, pp. 88-95, 2019.

[25] H. Chen, Y. Song, and X. Li, “A deep learning framework for identifying children with ADHD using an EEG-based brain network," Neurocomputing, vol. 356, no. 9, pp. 83-96, 2019.

[26] D. Cimr, F. Studnicka, H. Fujita et al., "Computer aided detection of breathing disorder from ballistocardiography signal using convolutional neural network," Information Sciences, vol. 541, pp. 207-217, 2020.

[27] F. Luo, P. Wang, G. Xu, and B. Zhang, "Crowd gathering abnormal prediction based on multi-scale convolutional neural network," Computer Engineering and Science, vol. 42, no. 12,10 pages, 2020.

[28] R. Wu, J. Li, and J. Qu, "Flight delay prediction model based on dual-channel convolutional neural network," Journal of Computer Applications, vol. 38, no. 7, 8 pages, 2018. 